\title{
Do Fielders Know Where to Go to Catch the Ball or Only How to Get There?
}

\author{
Peter McLeod \\ Oxford University
}

\author{
Zoltan Dienes \\ Sussex University
}

\begin{abstract}
Skilled fielders were filmed as they ran backward or forward to catch balls projected toward them from a bowling machine $45 \mathrm{~m}$ away. They ran at a speed that kept the acceleration of the tangent of the angle of elevation of gaze to the ball at 0 . This algorithm does not tell fielders where or when the ball will land, but it ensures that they run through the place where the ball drops to catch height at the precise moment that the ball arrives there. The algorithm leads to interception of the ball irrespective of the effect of wind resistance on the trajectory of the ball.
\end{abstract}

The everyday nature of the act of running to catch a ball can obscure the remarkable predictive ability that it requires. Figure 1 shows the trajectories of three balls projected at $45^{\circ}$ and approximately 22,24 , and $26 \mathrm{~m} / \mathrm{s}$ toward a stationary fielder $45 \mathrm{~m}$ away. They will land $5 \mathrm{~m}$ in front of, at, or $5 \mathrm{~m}$ behind the fielder, respectively. The solid line shows the trajectory of each ball in the first $840 \mathrm{~ms}$; the dashed line shows the rest of the flight. Within $840 \mathrm{~ms}$, most competent fielders would have started running forward for the ball on the lower trajectory and backward for the ball on the higher trajectory. ${ }^{1}$ Yet, the only difference between these two flights at this time is the difference between the longest and shortest solid lines. How is the fielder able to work out where to go from so little information?

Precise calculation of the trajectory is not possible because the essential ball flight parameters of projection angle, velocity, and wind resistance are available to the fielder only as, at best, crude estimates. Nor, given the infinite variation of trajectory, does it seem possible that learning to catch involves learning individual trajectories. An alternative is that an algorithm exists that links the visual information obtained from watching the ball's flight to a running speed that will bring the fielders to the correct place, irrespective of their starting position or the ball's trajectory. Learning to catch would involve the discovery of this algorithm.

This research was supported by a grant from the Oxford University Research and Equipment Fund.

We are indebted to Tommy McLeod for some of the crucial observations about the power of $\mathrm{d}^{2}(\tan \alpha) / \mathrm{dt}^{2}$. We thank Clare Mendham and Daniel Chambers for analyzing the films. We are grateful to a number of referees, particularly Michael McBeath, for their comments on earlier versions of this article.

Correspondence concerning this article should be addressed to Peter McLeod, Department of Experimental Psychology, Oxford University, South Parks Road, Oxford OX1 3UD England, or to Zoltan Dienes, Experimental Psychology, Sussex University, Brighton BN1 9QG England. Electronic mail may be sent via Internet to pdmcl@vax.ox.ac.uk or dienes@epvax.sussex.ac.uk.
Chapman (1968) analyzed the visual information available to a fielder watching a ball approaching in parabolic flight. He showed that if $\alpha$ is the angle of elevation of gaze from the fielder to the ball, then the acceleration of the tangent of $\alpha, \mathrm{d}^{2}(\tan \alpha) / \mathrm{dt}^{2}$, will be zero if, and only if, the fielder is standing at the place where the ball will land. He also suggested that this might be the basis of an interception algorithm. A fielder who starts at a place other than where the ball will land and runs at a constant velocity that keeps $\mathrm{d}^{2}(\tan \alpha) / \mathrm{dt}^{2}$ at zero will arrive at the correct place to make the catch at the same time as the ball.

However, because Chapman's (1968) work is based on the information provided by watching an object in parabolic flight, it is not clear what relevance it has to catching. Wind resistance ensures that objects in the real world do not follow parabolic trajectories. The departure from parabolic flight can be substantial at the speeds encountered in ball games. For example, Brancazio (1985) estimated that the effect of wind resistance on a well-hit baseball would be to reduce the horizontal distance traveled by up to $40 \%$ of the distance it would have achieved in parabolic flight. Furthermore, given identical projection angle and initial velocity, different objects follow different trajectories because of their different wind resistances. If catching involves learning an algorithm that links visual information to running speed, it must be one that works independently of the effect of wind resistance on trajectory.

Given Brancazio's (1985) analysis, one might be tempted to think that demonstrations of geometrical relationships that could form the basis of algorithms for intercepting balls in parabolic flight have no relation to real catching. However, a recent article has suggested otherwise. Michaels and Oudejans (1992) filmed two people running backward or forward to catch a ball. From the position of the catcher's

\footnotetext{
${ }^{1}$ In the current study, for example, the fielder started running in the correct direction within $840 \mathrm{~ms}$ for $74 \%$ of catches. Michaels and Oudejans (1992) also found that catchers started to run shortly after the ball appeared.
} 


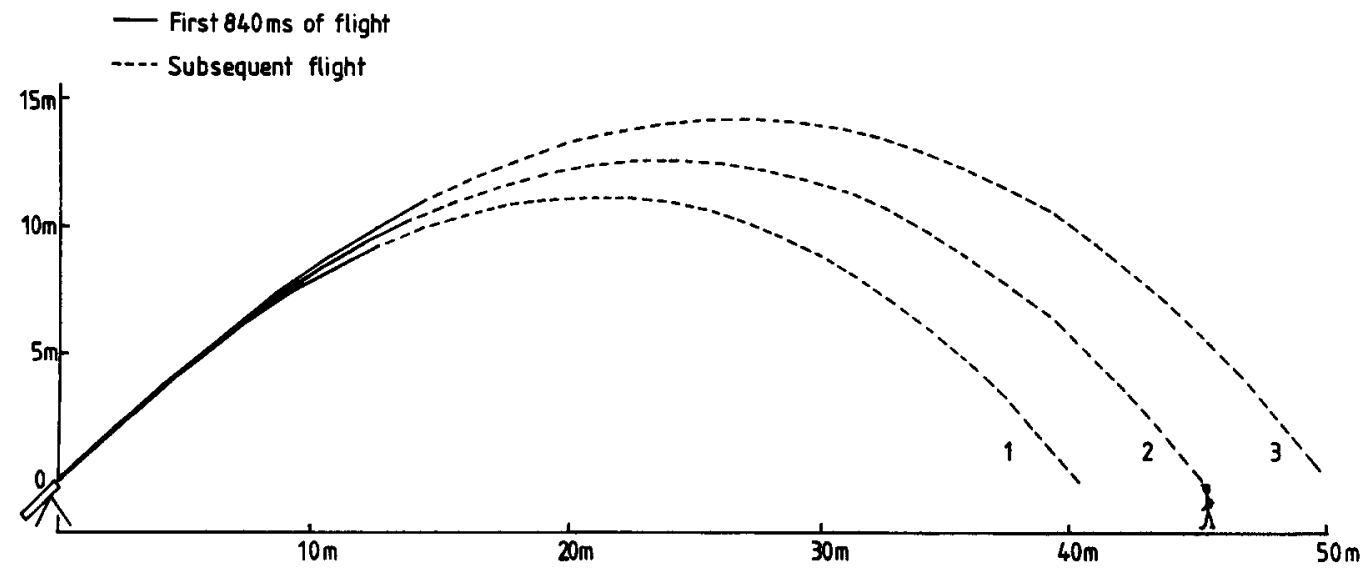

Figure 1. The trajectories of three balls projected at $45^{\circ}$ and a velocity (v) of $22.3,24.0$, and 25.7 $\mathrm{m} / \mathrm{s}$, respectively, toward a fielder $45 \mathrm{~m}$ away. They experienced a deceleration due to aerodynamic drag proportional to $\mathrm{v}^{2}$. The constant of proportionality was $0.007 \mathrm{~m}^{-1}$, a value typical of objects such as cricket balls (Daish, 1972).

head and of the ball, they were able to calculate the optic height of the ball throughout its flight. (The optic height is the position of the ball's image on an imaginary plane a fixed distance in front of the fielder's eye.) They showed that when fielders moved to make a catch, optic height increased with roughly constant velocity until just before the catch. Optic height is equivalent to the tangent of the angle of gaze, and constant velocity implies zero acceleration. Therefore, their result appears to offer support for Chapman's (1968) proposal that interception is ensured by running at a speed that maintains the acceleration of the tangent of the angle of gaze at zero. ${ }^{2}$

Before one concludes that Chapman (1968) was correct, we must elaborate on Michaels and Oudejans's (1992) result. First, their main experiment presented data from only 10 catches: 7 from one fielder and 3 from another. Second, they offered no statistical test of the linearity of the plots of optic height against time (i.e., of the claim that optic height increases at constant velocity). Third, although it is possible to fit a straight line by eye to the early parts of the plots of optic height against time for each catch, in the majority of catches there is a departure from linearity in the second half of the flight. ${ }^{3}$ They did not show whether Chapman's strategy will lead to interception (and that these deviations are unimportant) or whether the deviations are a necessary corrective process because Chapman's strategy does not actually get the fielder close enough to the ball to catch it (because of the effects of wind resistance). Finally, Michaels and Oudejans did not analyze the fielders' running velocity. Chapman's analysis requires not only that fielders should keep the velocity of optic height constant as they run but also that they find the constant running velocity at which this happens. If Chapman's analysis explains how fielders get to the right place at the right time, this condition must be met too.

The aim of the experiments reported here was to extend Michaels and Oudejans's (1992) analysis of whether running speed is controlled by an algorithm linked to some function of the angle of elevation of gaze to cover the four points above. We measured the running speed and the angle of elevation of gaze as skilled fielders ran to catch a ball. Successful interception usually requires the fielder to judge whether the ball is going to the left or the right as well as whether it is going to drop in front or behind. Visual cues that are available to make the left-right judgment have been identified (Regan, Beverley, \& Cynader, 1979; see also Regan, 1993; Regan \& Kanshal, 1994). Like Michaels and Oudejans, we considered the remaining problem of whether the fielder should move backward or forward to catch the ball. For simplicity, all our experiments involved balls projected in a vertical plane between the point of projection and the fielder so that the fielder did not have to move left or right. The algorithm that we show that fielders use in this situation works equally well in the more general case where the fielder must decide whether to move left or right as well as backward or forward.

\section{Experiment 1}

\section{Method}

\section{Participants}

Six skillful ball catchers participated. One was a professional soccer player, 1 played cricket at the professional level, and the remaining 4 were keen amateur cricket players. All were male.

\footnotetext{
${ }^{2}$ Optic height and the tangent of the angle of gaze are mathematically equivalent quantities, so the choice of one rather than the other may seem arbitrary. Angle of gaze is available directly to a fielder who looks at the ball; optic height is available directly to a fielder who maintains fixation on the point from which the ball was projected. Because fielders look at the ball when trying to catch it, not at the point of projection, it seems more appropriate to choose a function of the angle of gaze as the basis for the analysis.

${ }^{3}$ There is also a catastrophic departure, just before the catch, that occurs too late to be relevant to the question of how the fielder arrives at the right place to make the catch.
} 


\section{Catching}

The fielder stood approximately $45 \mathrm{~m}$ from a bowling machine, which projected a hard white ball into the air directly toward him at a projection angle of $45^{\circ}$. For different deliveries the speed was varied randomly over a range of about $20-25 \mathrm{~m} / \mathrm{s}$ so that the ball would unpredictably go over his head or fall short, with a range of about $\pm 10 \mathrm{~m}$ around his starting position. About 50 balls were fired at each fielder. He ran backward or forward or stayed where he was, trying to catch each ball.

\section{Measurement}

Fielder's position and velocity. Figure 2 shows a bird's-eye view of the experimental setup. The fielder ran backward and forward along an imaginary line between himself and the bowling machine to catch the ball. As he ran, he was tracked by a video camera. This had an electronic shutter, set to take images in $2 \mathrm{~ms}$, producing a blur-free image of the fielder. Beyond the fielder was a wall marked in units of $36 \mathrm{~cm}$. In frame-by-frame replay of the video, the position of the back of the fielder's head could be estimated to about $\pm 5 \mathrm{~cm}$ on the wall. Given the distance between camera, fielder, and wall, this uncertainty in measurement corresponded to an estimate of the position of the fielder accurate to about $\pm 3 \mathrm{~cm}$. The fielder's position was sampled every $120 \mathrm{~ms}$. The positional estimates from the frame-by-frame analysis were smoothed with a Hanning window, each position being recalcu-

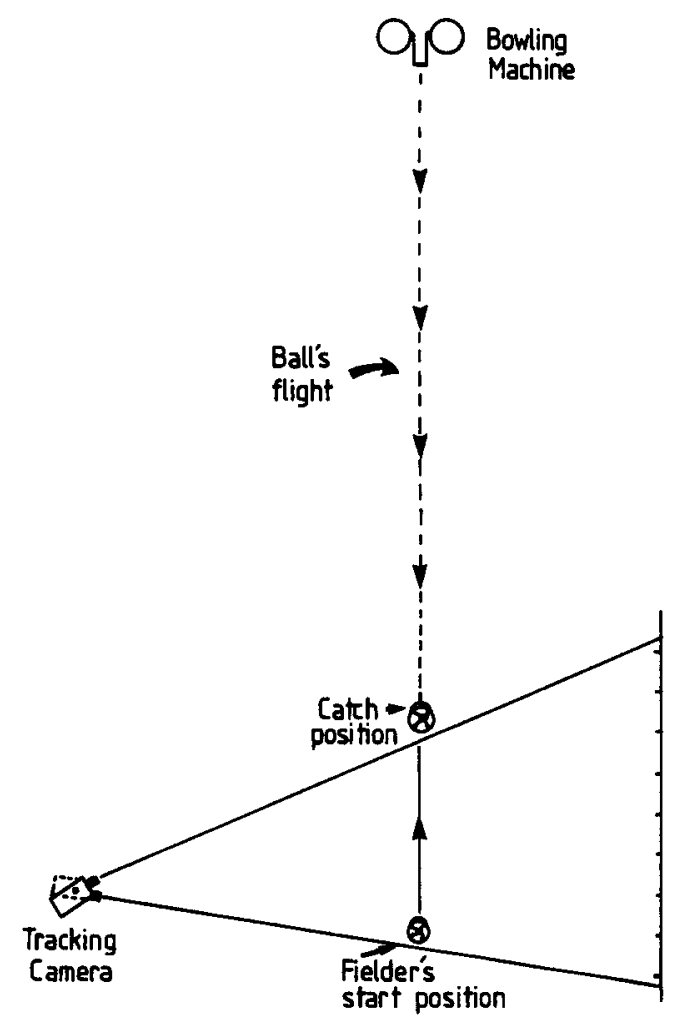

Figure 2. The experimental setup as viewed from above. As the fielder ran to catch the ball, he was tracked by the video camera. Given that he was running in a straight line toward or away from the bowling machine, his real position could be calculated from the position that he had reached against the structured background. lated as half of itself plus one quarter of each of its immediate neighbors. The smoothed position estimates were differentiated to give the fielder's velocity.

Position of the ball. The position of the ball in flight was not recorded on video (except in the final frames when the fielder was about to catch it), but it was possible to analytically estimate its position throughout the flight. The initial velocity and projection angle of the ball were known. The distance it traveled was known because its position was recorded on the video as it appeared against the structured background just before it was caught. The flight duration was known (to within $\pm 40 \mathrm{~ms}$, the duration of the video frame) because a marker appeared on the video at the moment the ball left the bowling machine and the moment when the ball was caught was recorded on the video.

These four values were used to compute the trajectory of the ball, assuming parabolic flight modified by an aerodynamic drag factor, proportional to the square of the ball's velocity. The value of the drag factor was estimated by finding the value that gave the lowest summed mean squared difference between observed and predicted values of flight distance and flight time. With the best fit value for wind resistance, the errors were about $3 \%$ in estimating flight duration and $1 \%$ in estimating flight extent. The values we obtained were similar to that given by Daish (1972) for a cricket ball. Given drag, initial velocity, and projection angle, it was possible to calculate the height of the ball and its distance from the bowling machine at any time during the flight. (A detailed account of the method is given in the article by Dienes and McLeod, 1993.)

Angle of gaze. The initial positions of the fielder and the ball were known. The position of the fielder after Time $t$ was measured from the video, and the position of the ball after Time $t$ was calculated as described above. The angle of gaze from the fielder to the ball follows directly.

\section{Results}

\section{Running Speed}

The left side of Figure 3 shows six typical examples of running data from 1 fielder. His velocity as he ran to catch the ball is plotted against time, each curve ending at the time when the ball was caught. Each curve is labeled with the distance he ran, a negative sign indicating that he ran backward. (All fielders showed qualitatively similar patterns. Combining data to show average running patterns, or to compute the variance of the running patterns, is problematic because the fielders ran different distances and paused for different lengths of time before starting to run.)

Figure 3 demonstrates two effects shown by all fielders. ${ }^{4}$ First, they were always moving when they caught the ball (except when they had no more than 1-2 m to cover to make the catch). (Although Michaels and Oudejans, 1992, did not comment on this effect, it can be seen from their Figure 4 that they found the same result. In all of the catches where the fielder moved more than about $2 \mathrm{~m}$, she was moving

\footnotetext{
${ }^{4}$ The fielder shown in Figure 3 also showed one effect not shown by all fielders. He always took one or two steps forward before moving backward. This may reflect the fact that in cricket, deep fielders (i.e., ones fielding at some distance from the bat) usually walk toward the batsman as the ball is bowled. However, we do not know why some of our participants did this and others did not.
} 


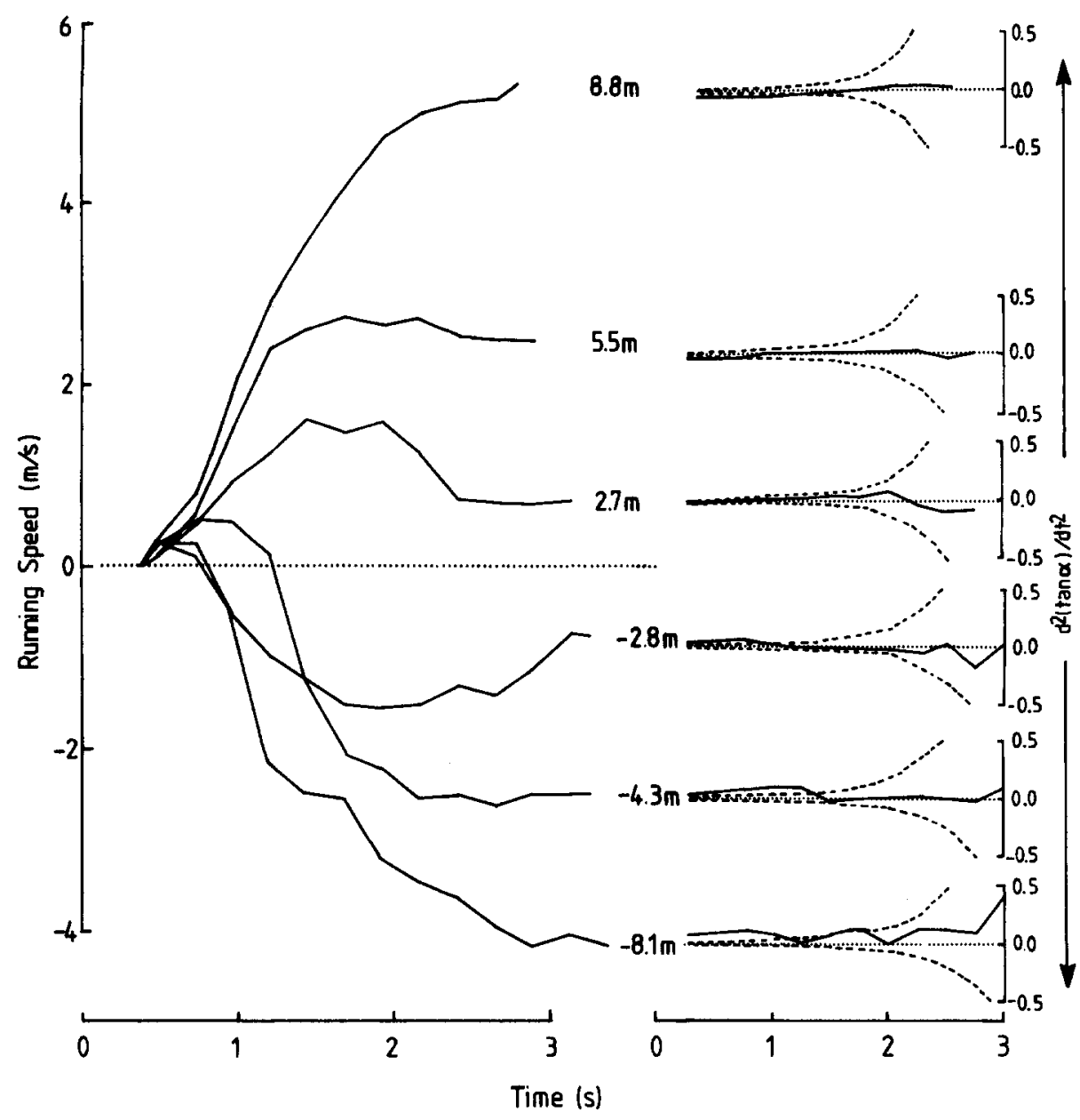

Figure 3. The left panel shows the fielder's running speed as a function of time as he ran forward to catch balls $8.8,5.5$, or $2.7 \mathrm{~m}$ in front of his starting position or as he ran backward to catch balls $2.8,4.3$, or $8.1 \mathrm{~m}$ behind his starting position. (He actually started running about $0.5 \mathrm{~s}$ after the ball appeared. His velocity is shown as greater than zero slightly earlier as a result of the smoothing algorithm being applied to the raw position data before the velocity was calculated.) The right panel shows $\mathrm{d}^{2}(\tan \alpha) / \mathrm{dt}^{2}$, where $\alpha$ is the angle of elevation of gaze from the fielder to the ball. The solid line shows the value that we calculated the fielder saw as he ran with the velocity shown on the left of the figure; the dashed lines show the value that he would have seen if he had run at a constant velocity, which was either too fast or too slow so that he missed the ball by $2 \mathrm{~m}$.

when she caught the ball.) Thus, the fielder does not run to the point where the ball will fall and then wait for it but rather runs through the point where the ball will fall at the exact moment that it arrives there. A fielder who knew where the ball was going to fall would presumably run to that point and wait for it to arrive. So, it is possible that the fielder does not know where the ball will fall when running.

This possibility suggests a solution to the problem, illustrated by Figure 1, that when the fielder starts to run, there appears to be insufficient information to work out where the ball will land. This lack of information is paradoxical if the fielder is assumed to know where the ball will land when starting to run. However, if it turns out that the fielder does not know where the ball will land, the problem disappears. There may be sufficient information in the first few hundred milliseconds of the ball's flight to tell the fielder in which direction to start running, even though there is not enough to tell where or when the ball will land.

The second point made by Figure 3 is that the running patterns for different distances had nothing in common. Long runs involved continuous acceleration, medium distances involved acceleration and then constant velocity, and short distances involved acceleration and then deceleration. The requirement of Chapman's (1968) analysis, that fielders should run at constant velocity, did not hold. A variant of Chapman's proposal that might allow for this has been put forward by Babler and Dannemiller (1993). They suggested that the fielder starts running at the constant velocity that zeros optic acceleration, as Chapman suggested. When, owing to the nonparabolic flight, optic acceleration starts to 
increase or decrease, the fielder finds a new velocity that zeros it, and so on, repeatedly throughout the flight. The idea, in effect, is that a nonparabolic flight could be approximated by a series of roughly parabolic sections. Thus, even a fielder following Chapman's method might not run at the same speed throughout the catch. Although Babler and Dannemiller's proposal seems intuitively reasonable, they gave no proof of the effectiveness of this algorithm. Nor does it resolve the problem, inherent in Chapman's strategy, of how the fielder finds the constant running speed that results in zero optic acceleration. Whether the strategy could work in principle, it is difficult to see either of the catches at the longest distance, with continuous acceleration throughout the flight, offering support even for this modified version of Chapman's proposal.

\section{Angle of Gaze}

The right side of Figure 3 shows the value of $d^{2}(\tan \alpha) / \mathrm{dt}^{2}$ (where $\alpha$ is the angle of elevation of gaze from the fielder to the ball) that the fielder would have seen as he watched the ball during each run up to $240 \mathrm{~ms}$ before the catch was made. ${ }^{5}$ It appears that he waited for about $0.5 \mathrm{~s}$ and then started to run, accelerating until he reached a speed where $\mathrm{d}^{2}(\tan \alpha) / \mathrm{dt}^{2}=0$. Then, he modulated his speed up to the point of catching the ball. If he was running forward, he ran faster if $\mathrm{d}^{2}(\tan \alpha) / \mathrm{dt}^{2}$ became negative and more slowly if it became positive, and if he was running backward, vice versa.

Clearly, $d^{2}(\tan \alpha) / \mathrm{dt}^{2}$ is maintained close to zero, but is it close enough to ensure that the ball is caught? This can be assessed by considering what the value would be if the fielder just failed to reach the ball. Given that arm reach is about $1 \mathrm{~m}$ and allowing for a jump or lunge as the ball is caught, the fielder would just be unable to catch the ball if he was about $2 \mathrm{~m}$ away from the ball when it reached catching height. The dashed lines show the value of $d^{2}(\tan$ $\alpha) / \mathrm{dt}^{2}$ that the fielder would have observed had he run at a constant speed that would have taken him to a point either $2 \mathrm{~m}$ short of or $2 \mathrm{~m}$ beyond the place where the ball would fall. It can be seen that in every flight the value of $d^{2}(\tan$ $\alpha) / \mathrm{dt}^{2}$ actually experienced by the fielder would take him to less than $2 \mathrm{~m}$ from the place where the ball would fall. The deviations of $d^{2}(\tan \alpha) / d^{2}$ from zero were insufficient to prevent the fielder from intercepting the ball.

To test the claim that $\mathrm{d}^{2}(\tan \alpha) / \mathrm{dt}^{2}=0$, we plotted regression lines of $\mathrm{d}^{2}(\tan \alpha) / \mathrm{dt}^{2}$ against time, from the time when the fielder started running until $240 \mathrm{~ms}$ before he made the catch. For a random sample of 15 successful catches by the fielder whose data are shown in Figure 3, the medians of the absolute values of the intercepts and the slopes of these regression lines (i.e., taking the median of the absolute value and ignoring the sign) were $0.02 \mathrm{~s}^{-2}$ (signed range from -0.07 to 0.07 ) and $0.02 \mathrm{~s}^{-3}$ (signed range from -0.04 to 0.10 ), respectively. In only one flight was the value of either slope or intercept reliably different (at the 5\% level) from zero. A random sample of 27 successful catches from the other fielders showed a similar result: The median values of the absolute intercepts and slopes were $0.04 \mathrm{~s}^{-2}$ (signed range from -0.08 to 0.09 ) and $0.04 \mathrm{~s}^{-3}$ (signed range from -0.04 to 0.09 ), respectively. Only 2 of the catches gave either a slope or an intercept reliably different from zero.

\section{Experiment 2}

Experiment 1 showed that when the fielder had less far to run he ran more slowly, rather than running to the point where the ball would fall and waiting for it (see the runningspeed data in Figure 3). If he knew where to go, running more slowly and arriving just in time to catch the ball would seem a pointlessly risky strategy. Why not go to the right place and wait? However, if the fielder does not know where the ball will land but is following a strategy that will get him to the right place at the right time, it is inevitable that he will run more slowly if he has more time. Experiment 2 was a direct test of this possibility.

\section{Method}

The projection angle of the ball was increased to $64^{\circ}$. With an initial projection velocity of $24 \mathrm{~m} / \mathrm{s}$, it now fell about $36 \mathrm{~m}$ from the bowling machine, a distance similar to the balls projected at $45^{\circ}$ and $20 \mathrm{~m} / \mathrm{s}$ in Experiment 1 . However, because the trajectory was higher, the ball took longer to get there. If the fielder knew where he was going, he could go there and wait for the ball on the higher, longer trajectory. But if he was following a strategy that would lead him to arrive at the same time as the ball, he would run more slowly.

\section{Results}

\section{Running Speed}

Figure 4 (upper panel) shows the fielder's speed for six different catches as he ran 8-10 $\mathrm{m}$ to catch the balls on the two different trajectories. The upper curve shows his mean speed for three catches when the ball was projected at $45^{\circ}$; the lower curve shows his mean speed for three catches when the ball was projected at $64^{\circ}$. The bars show the range of speed over the three runs. It can be seen that the separation of the curves representing the means is an accurate reflection of the individual catches because there is no overlap between the individual curves from the two groups. The curves end at the point where the fielder made the catch. In both cases, he arrived at the point where the ball fell at the same time as the ball (i.e., he had a positive velocity at the moment that he made the catch). With the

\footnotetext{
${ }^{5}$ Like Michaels and Oudejans (1992), we usually found a catastrophic jump in $\mathrm{d}^{2}(\tan \alpha) / \mathrm{dt}^{2}$ over the last two data points. The sudden change in $\alpha$ that gave rise to this effect was caused, at least in part, by the fact that the ball was not caught at the point that was taken to represent the origin of the fielder's angle of gaze. The sudden change in $\alpha$ may well have had some role in the terminal reach adjustment immediately before the catch, but it occurred too late to be relevant to the question of how the fielder got close enough to the ball to make the catch, so we ignored it.
} 


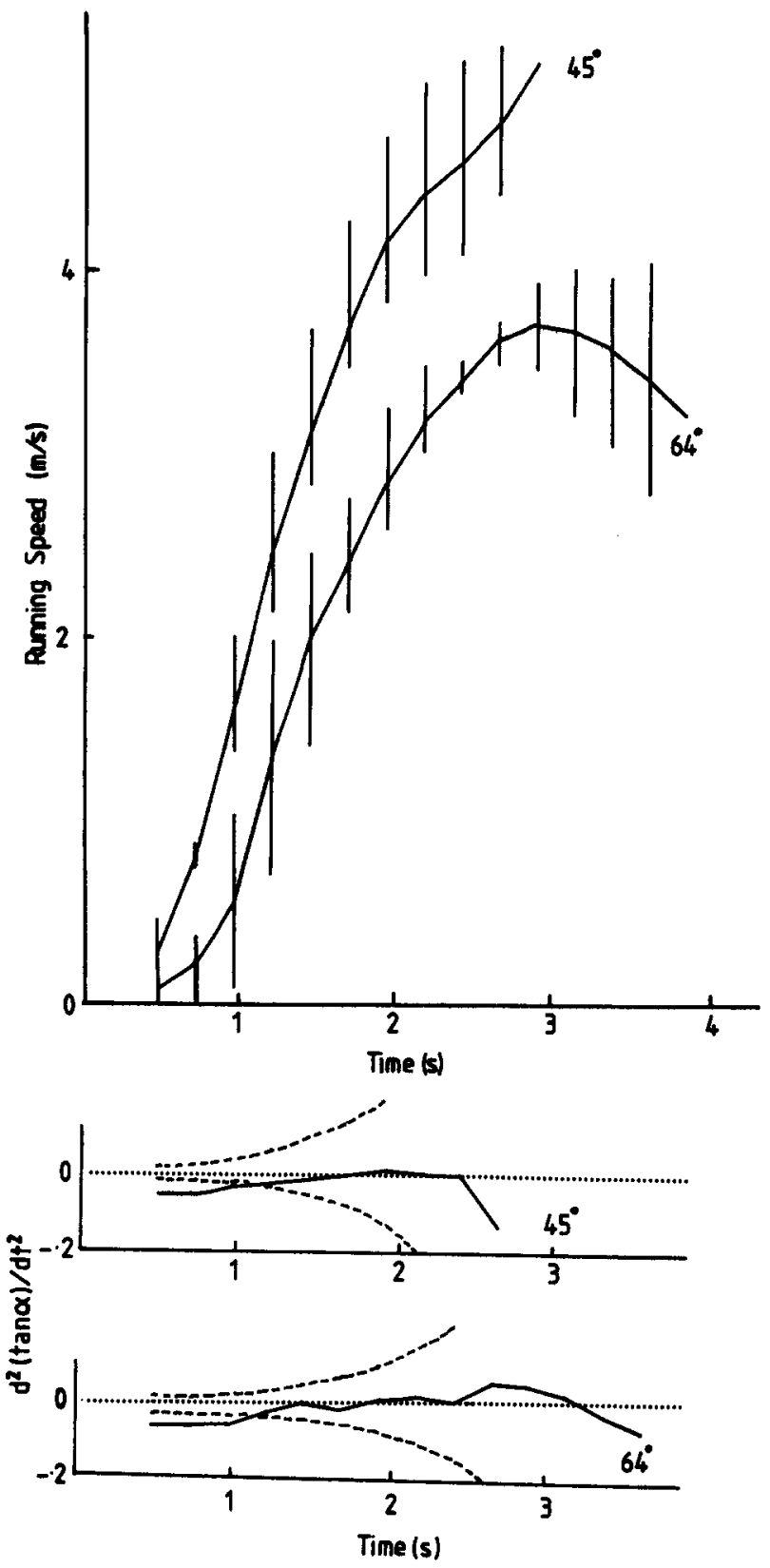

Figure 4. The fielder ran to catch balls landing $8-10 \mathrm{~m}$ in front of him. The ball had an initial projection angle of $45^{\circ}$ (flight time about $3 \mathrm{~s}$ ) or $64^{\circ}$ (flight time about $4 \mathrm{~s}$ ). The top panel shows the fielder's running speed as a function of time as he ran to catch the ball. Each curve is the mean of three runs. The bars show the range of velocities across the three runs. In the bottom panel, the solid lines show $\mathrm{d}^{2}(\tan \alpha) / \mathrm{dt}^{2}$ for each run, and the dashed lines show what the value would have been if he had run at constant velocity to a point $2 \mathrm{~m}$ short of or $2 \mathrm{~m}$ beyond the point where the ball fell.

longer trajectory, he ran more slowly. This finding supports the conclusion of Experiment 1: The strategy he used got him to the right place at the right time. However, it does not appear to tell him where that place is in advance.

\section{Angle of Gaze}

Figure 4 (lower panel) plots the value of $\mathrm{d}^{2}(\tan \alpha) / \mathrm{dt}^{2}$ for each run. The solid lines are the values of $d^{2}(\tan \alpha) / \mathrm{dt}^{2}$ that the fielder would have seen as he ran (averaged over the three flights). The dashed lines show what he would have seen if he had run at constant velocity to a point $2 \mathrm{~m}$ short of or $2 \mathrm{~m}$ beyond the place where the ball fell.

The upper panel of Figure 4 shows that two quite different running patterns were produced to get the fielder to the same place when the ball's trajectory was changed. What they have in common is that the fielder ran at a speed that kept $\mathrm{d}^{2}(\tan \alpha) / \mathrm{dt}^{2}$ close to zero. Regression lines of $\mathrm{d}^{2}(\tan$ $\alpha) / \mathrm{dt}^{2}$ against time, plotted for the individual catches, show only one catch for which either intercept or slope was reliably different from zero. For the $45^{\circ}$ projection angle, the medians of the absolute values of the intercepts and the slopes were $0.04 \mathrm{~s}^{-2}$ (range from -0.07 to 0.04 ) and 0.02 $\mathrm{s}^{-3}$ (range from 0.02 to 0.04 ), respectively. For the $64^{\circ}$ projection angle, the medians of the absolute values of the intercepts and the slopes were $0.07 \mathrm{~s}^{-2}$ (range from -0.07 to 0.09 ) and $0.04 \mathrm{~s}^{-3}$ (range from -0.04 to 0.05 ), respectively.

\section{Conclusion}

The fact that the fielders did not use spare time to run to the place where the ball would fall and wait suggests that they did not know where it would fall. However, the experiments did not directly test the fielders' knowledge of where the ball would fall. It can be concluded that the algorithm fielders use to intercept the ball is one that ensures they arrive at the right place at the right time but does not tell them where or when that is. Whether fielders know where the ball will land but choose not to use this information as they run to catch it is a possibility that awaits further experimentation.

\section{Missing the Ball}

We claim that fielders use the sign of $\mathrm{d}^{2}(\tan \alpha) / \mathrm{dt}^{2}$ as the input to a servo that controls running speed. When running backward, they speed up when it is positive and slow down when it is negative, and when running forward, vice versa. This strategy is illustrated by seeing what happens when a fielder fails to run fast enough to catch the ball. Figure 5 compares two catches where the ball landed in roughly the same place, about $6 \mathrm{~m}$ behind the starting position of the fielder. In one case (open circles), he successfully caught the ball; in the other (filled circles), he started running backward too slowly, and the ball went over his head, just out of reach of his outstretched hand.

The upper panel of Figure 5 shows his velocity. For the successful catch, he initially accelerated backward, eventually decelerated, and maintained an approximately constant velocity until he made the catch. In the unsuccessful case, he was slower to start and accelerated more slowly but 

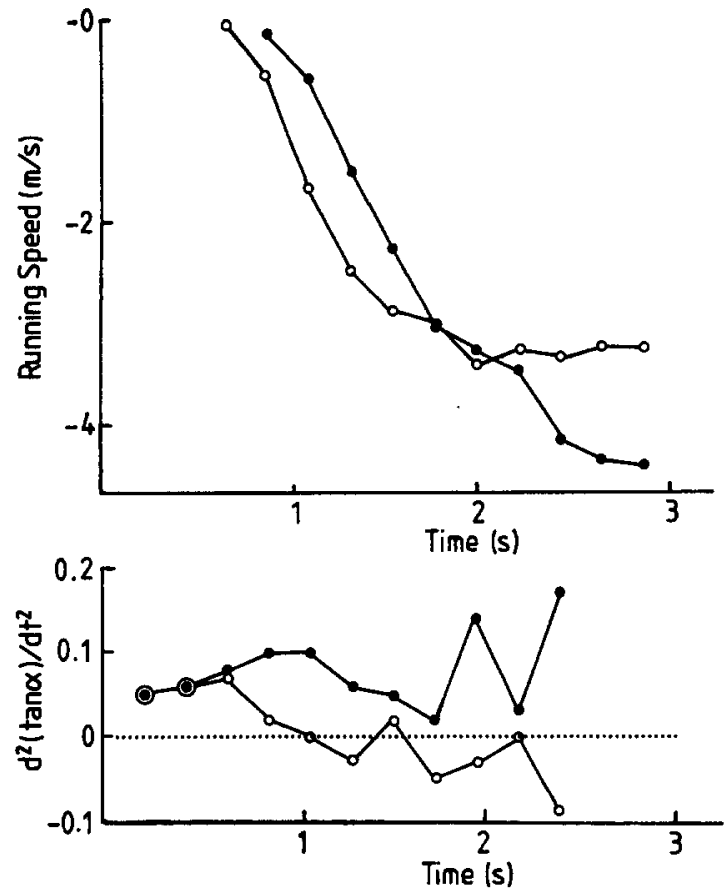

Figure 5. Velocity and $\mathrm{d}^{2}(\tan \alpha) / \mathrm{dt}^{2}$, as in Figure 3, for two catches where the fielder ran backward. Open circles represent that he caught the ball; filled circles represent that the ball went over his head.

continued to accelerate throughout the flight of the ball. Why did he produce these different running patterns?

The lower panel of Figure 5 shows $\mathrm{d}^{2}(\tan \alpha) / \mathrm{dt}^{2}$ for each flight. In both cases, the value started positive (because the ball was going over the fielder's head) and increased (because the fielder was initially stationary). Once the fielder started to run in the appropriate direction, the value came down. For the successful catch (open circles), it reached zero, so he stopped accelerating. For the unsuccessful catch (filled circles), $\mathrm{d}^{2}(\tan \alpha) / \mathrm{dt}^{2}$ remained positive throughout the flight, so the fielder continued to accelerate but to no avail. He started too late and could not run fast enough to intercept the ball.

\section{General Discussion}

We manipulated the time that a fielder had to run for a catch in two ways. We made the ball fall nearer to or farther from him (Experiment 1); we made it land at the same place, but it took longer to get there (Experiment 2). In both experiments there were apparently complex changes in the fielder's running speed (see Figures 3 and 4). But in all cases one thing remained constant: $\mathrm{He}$ ran at a speed that kept the acceleration of the tangent of the angle of elevation of gaze close to zero. This result was predicted by Chapman (1968). But we know that Chapman's analysis cannot be correct. First, it is based on the information available from watching a parabolic flight. The balls in this experiment were thrown sufficiently fast to have departed considerably from parabolic flight. ${ }^{6}$ Second, Chapman's algorithm assumes that fielders run at constant velocity. Figures 3 and 4 show that typically they do not do so.

A possible resolution is Babler and Dannemiller's (1993) suggestion that these two problems are linked. They proposed that as the flight departs from parabolic, the fielders adjust their running velocity, finding a series of new values of constant velocity throughout the flight successively zeroing out optic acceleration. But a fundamental problem still remains. To discover that Chapman's (1968) strategy works requires people to view parabolic flights (which they never, in fact, experience) while running at one particular constant velocity (or set of constant velocities, if we adopt Babler \& Dannemiller's, 1993, argument). The value of the constant velocity (or set of velocities) would be different for every flight they experienced. The implausibility of anyone ever discovering that Chapman's algorithm led to interception, coupled with the fact that most children learn to catch just by watching balls in flight, suggests the need for a different approach.

\section{How to Intercept a Ball}

Consider Figure 6a. A ball is falling, watched by a fielder. The angle of elevation of gaze from the fielder to the ball is $\alpha$. The height of the ball above the ground is $y$, and the horizontal distance from the fielder to the ball is $\mathrm{x}$. The requirement for intercepting the ball before it hits the ground is simple: As $y \rightarrow 0, x \rightarrow 0$. That is, as the ball drops to the ground, the fielder reaches the place where it drops. This is illustrated in Figures $6 \mathrm{~b}$ and $6 \mathrm{c}$, where the fielder closes in on the ball $(x \rightarrow 0)$ as it falls $(y \rightarrow 0)$. If both $x$ and $y \rightarrow 0$ together, $\alpha$ will always be positive but less than $90^{\circ}$, that is, $0^{\circ}<\alpha<90^{\circ}$. If the fielder fails to intercept the ball, one of two things must happen: Either it falls in front of him, in which case $\alpha \leq 0^{\circ}$ (Figure 6d) or it goes over his head, in which case $\alpha \geq 90^{\circ}$ (Figure 6e). ${ }^{7}$

The conditions for intercepting or, alternatively, failing to reach the ball are surprisingly simple: If the fielder runs at a speed that ensures that the angle of gaze is greater than $0^{\circ}$ but less than $90^{\circ}$ throughout the flight, the ball will be intercepted. If the angle of gaze reaches either $0^{\circ}$ or $90^{\circ}$, the

\footnotetext{
${ }^{6}$ For example, the ball projected at $64^{\circ}$ and $24 \mathrm{~m} / \mathrm{s}$ in Experiment 2 traveled $36 \mathrm{~m}$. In parabolic flight, it would have traveled about $46 \mathrm{~m}$.

${ }^{7}$ Strictly, this is true only if balls can only be caught just in front of the eyes-the point from which the angle of elevation of gaze is measured. Of course, fielders can stretch their arms forward and catch a ball a few feet in front of them, despite the fact that $\alpha$ has gone to $0^{\circ}$, or catch one going just over their head, when $\alpha$ will have reached $90^{\circ}$. However, in cricket at least, fielders prefer to catch a ball that has been hit high in the air just in front of their eyes (see, e.g., Richards \& Murphy, 1988, p. 127). They stretch their arms out to catch the ball as a last moment adjustment only if they have failed to get to the right place. We based our analysis on the assumption that fielders are endeavoring to run to the optimum place for catching, realizing that some other algorithm also exists to control the arm movements that allow for a correction just as the ball arrives if they fail to reach the ideal point.
} 
a

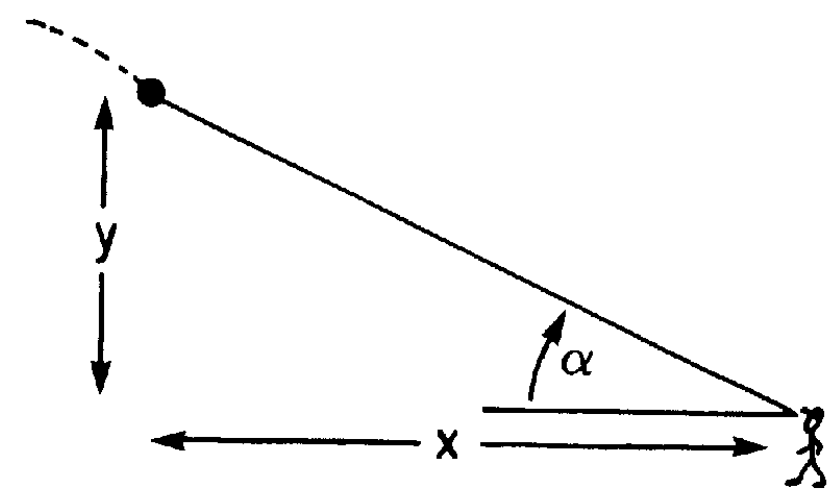

b

C

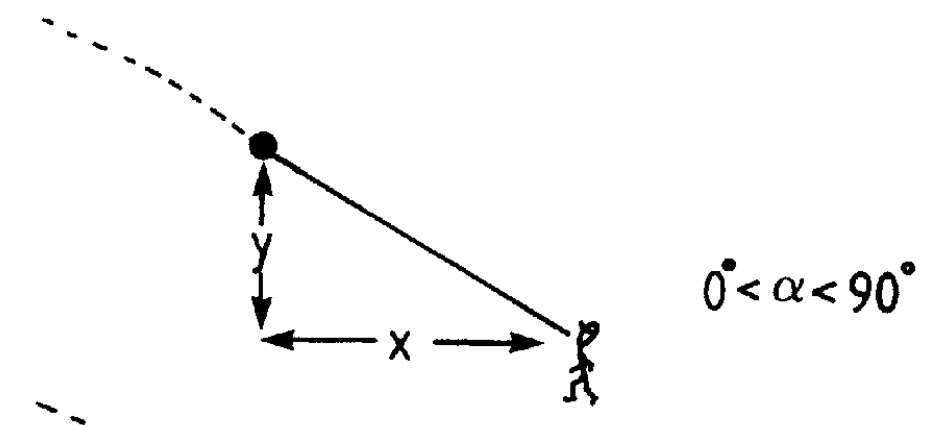

d

e
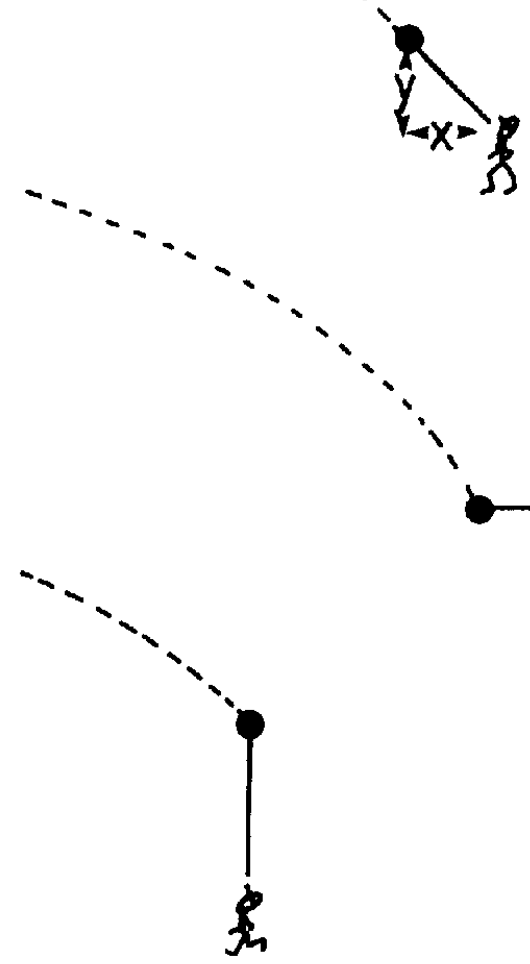

$0^{\circ}<\alpha<90^{\circ}$

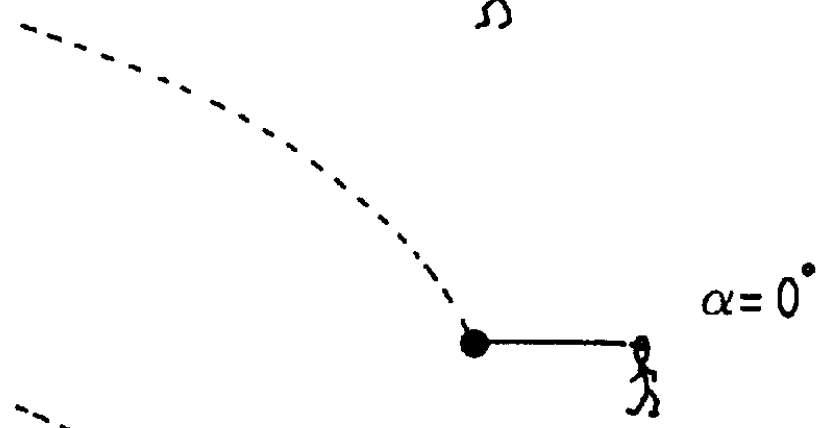

Figure 6. The conditions for intercepting or missing the ball. (a) $\alpha$ is the angle of elevation of gaze as the fielder watched the ball. If the fielder ran at a speed that kept $\alpha$ positive but less than $90^{\circ}$ as the ball fell ( $\mathrm{b}$ and $\mathrm{c}$ ), it would be intercepted. If $\alpha$ fell to $0^{\circ}$ or reached $90^{\circ}$, the ball would not be intercepted because it would have fallen in front (d) or gone over the fielder's head (e). 
ball will be missed (with the exception mentioned above of a ball within reach of an outstretched hand). This is illustrated in Figure 7, which shows the angle of gaze for the fielder in Figure 1 watching the flight of the balls on the three trajectories shown there. (Figure 7 shows the angle of gaze for a stationary fielder. If the fielder ran to catch the ball, unsuccessfully in the case of the ball landing in front of or behind him or successfully in the case of the ball landing in his hands, the picture would be qualitatively similar.) The angle of elevation of gaze, $\alpha$, starts at zero in each case and increases as the fielder watches the ball rising in the air. For the ball that will land in his hands, $\alpha$ continues to increase throughout the flight. But the rate of increase slows down as the flight progresses, and $\alpha$ never reaches $90^{\circ}$. For the ball that will fall in front of him, $\alpha$ reaches a maximum, starts to decrease, and accelerates back toward zero. For the ball that will go over his head, $\alpha$ accelerates throughout the flight, reaching $90^{\circ}$ as the ball passes over his head. Although the exact way that $\alpha$ changes throughout the flights will, of course, vary with angle and velocity of projection and the wind resistance of the object, this general pattern is shown for all flights. For any flight that the fielder misses, $\alpha$ reaches either $0^{\circ}$ or $90^{\circ}$; for any flight that is intercepted, $0^{\circ}<\alpha<90^{\circ}$ throughout the flight.

\section{Why Running so That $d^{2}(\tan \alpha) / d t^{2}=0$ Results in the Ball Being Intercepted}

The reason why running at a speed that keeps $d^{2}(\tan$ $\alpha) / \mathrm{dt}^{2}=0$ leads to interception can be understood by considering the usual case where the fielder starts to run while the angle of elevation of gaze is increasing; $\alpha$, and thus $\tan \alpha$, will be positive and increasing when the fielder starts. If the fielder runs at a speed that keeps $\mathrm{d}^{2}(\tan \alpha) / \mathrm{dt}^{2}$ $=0, \tan \alpha$ must be positive and finite at the end of the flight (i.e., $0<\tan \alpha<\infty$ ). Because $\tan 0^{\circ}$ is 0 and $\tan 90^{\circ}$ is $\infty$, it follows that $\alpha$ will lie between $0^{\circ}$ and $90^{\circ}$ at the end of the flight. But this is the condition for intercepting the ball. Therefore, if the fielder can run fast enough to keep $d^{2}(\tan$ $\alpha) / \mathrm{dt}^{2}=0$, the ball will be intercepted. ${ }^{8}$

\section{Chapman and $d^{2}(\tan \alpha) / d t^{2}$}

Chapman (1968) demonstrated a curious geometrical relationship. If a fielder runs toward the place where a ball in parabolic flight will fall at the constant velocity that will cause him or her to arrive at the same time as the ball, $d(\tan$ $\alpha) / \mathrm{dt}$ will be constant throughout the run. Because, by definition, Chapman's fielder successfully intercepts the ball, this observation has sometimes been seen as offering a solution to the problem of how to catch the ball. The fact that Michaels and Oudejans (1992; and now we) have shown that $\mathrm{d}(\tan \alpha) / \mathrm{dt}$ is constant, that is, $\mathrm{d}^{2}(\tan \alpha) / \mathrm{dt}^{2}=0$, as fielders run is seen as support for this belief.

Chapman's (1968) observation is geometrically correct, but it does not offer a solution to the problem facing the fielder. To run at constant velocity to the interception point requires the fielder to know, when he or she starts, what distance must be run in what length of time. Running at constant velocity thus requires the fielder to know where and when the ball will land. So Chapman's observation does not lead to a solution to the problem of intercepting the ball because it requires the fielder to know the answer (i.e., where and when the ball will land) before it can be implemented.

An alternative way of construing Chapman's (1968) observation into a solution of the problem facing the fielder is to turn it round. If the fielder runs so as to keep $\mathrm{d}(\tan \alpha) / \mathrm{dt}$ constant at one particular value, velocity will turn out to be the one constant velocity that produces interception. The problem with this is that the crucial value of $\mathrm{d}(\tan \alpha) / \mathrm{dt}$ would be different for every ball trajectory and every starting position. There is no reason to believe that the fielder could know all these values. (There is also the empirical problem for this approach that people do not run at constant velocity!)

Although we agree with Chapman (1968; and with Michaels \& Oudejans, 1992) that a crucial part of the fielder's strategy involves keeping $\mathrm{d}^{2}(\tan \alpha) / \mathrm{dt}^{2}$ at zero, our interpretation of what the fielder is doing is entirely different from theirs. We believe that keeping $\mathrm{d}(\tan \alpha) / \mathrm{dt}$ constant leads to interception because it keeps $\alpha$ between $0^{\circ}$ and $90^{\circ}$, not because it produces the one constant running velocity that will produce interception. Chapman showed that interception would occur if the fielder kept the one value of $d(\tan$ $\alpha) / \mathrm{dt}$ constant that produces constant running speed. In fact, a fielder who keeps any value of $\mathrm{d}(\tan \alpha) / \mathrm{dt}$ constant will intercept the ball (see Dienes \& McLeod, 1993), but only the "Chapman" value for any given flight will result in a constant running velocity.

Once one realizes that any constant value of $\mathrm{d}(\tan \alpha) / \mathrm{dt}$ will produce interception, the problem for the fielder, inherent in Chapman's (1968) strategy, of knowing the crucial value of $\mathrm{d}(\tan \alpha) / \mathrm{dt}$ disappears. The fielder, having sighted the ball for long enough to have generated a value of $d(\tan$ $\alpha) / d t$ (which will be different for different trajectories), simply has to run at a speed that keeps it constant. This speed will usually vary during the run. If the fielder waits a little longer before starting to run, the initial value of $d(\tan$ $\alpha) / \mathrm{dt}$ will change, and a different running pattern will result. But the ball will still be intercepted if the fielder can run fast enough to keep $d(\tan \alpha) / d t$ constant.

\section{Alternative Strategies}

Interception will occur provided the fielder ensures that $0^{\circ}<\alpha<90^{\circ}$ throughout the ball's flight. The strategy that skilled fielders appear to use, keeping $\mathrm{d}^{2}(\tan \alpha) / \mathrm{dt}^{2}$ at zero, has been shown to be a very effective strategy for doing this. It works for any trajectory and whatever the time the fielder starts running, provided the ball has a horizontal velocity

\footnotetext{
${ }^{8}$ Surprisingly, this strategy usually leads to interception even if $\mathrm{d}(\tan \alpha) / \mathrm{dt}<0$ (i.e., the ball is already falling) when the fielder starts to run. Proof of this and an analysis of the conditions when the strategy will not work can be found in Dienes and McLeod (1993).
} 


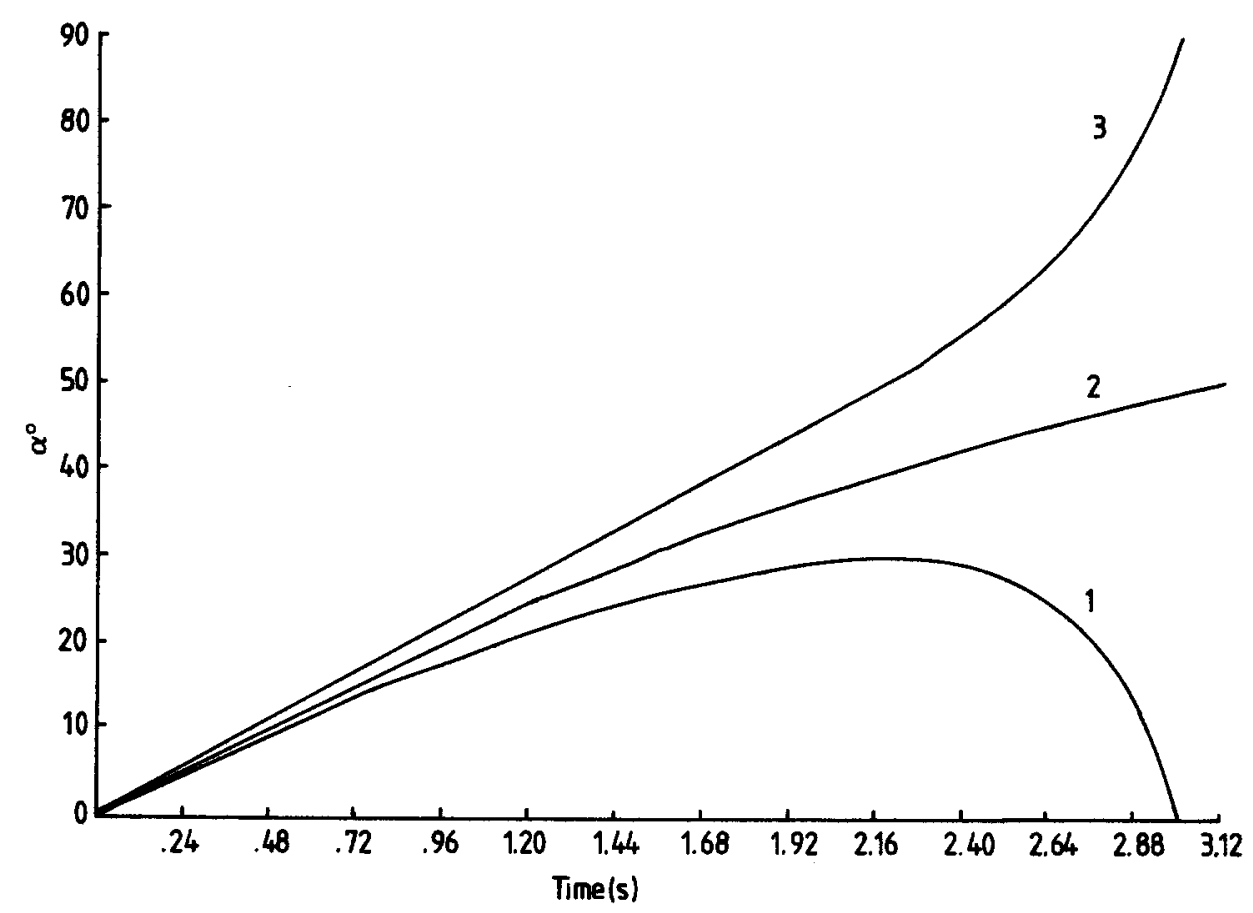

Figure 7. How the angle of elevation of gaze, $\alpha$, varied with time as the stationary fielder in Figure 1 watched the flights of the ball falling in front (1), at (2), or going overhead (3).

component toward the fielder (Dienes \& McLeod, 1993). However, it might seem unnecessarily complex. We exam- ined two strategies that might seem simpler and thus more plausible but showed that they were not as effective.

\section{Constant $\alpha$}

The simplest strategy for ensuring that $\alpha$ lies between $0^{\circ}$ and $90^{\circ}$ is to maintain a constant $\alpha$. Maintaining a constant $\alpha$ is equivalent to keeping $\mathrm{d}(\tan \alpha) / \mathrm{dt}$ rather than $\mathrm{d}^{2}(\tan$ $\alpha) / \mathrm{dt}^{2}$ at zero.

In games like cricket and baseball, the ball is seldom in the air for more than a few seconds. So one limit on the ability of fielders to catch the ball is set by how far they can run while the ball is in the air. The sooner fielders start running in the right direction, the farther they will be able to run in the time available. Thus, the most effective strategy is the one that gets the fielder running in the right direction, at the highest speed, soonest.

Figure 8 shows the flight of a ball that will fall in front of the fielder. To intercept it, the fielder must run forward. A fielder who tries to ensure interception by maintaining a constant angle of gaze (see Figure 8a) will start by running in the wrong direction. As the ball descends, the fielder will then have to change direction, recovering lost ground before starting to make any progress from the original starting position to the place where the ball will land. Obviously a more effective strategy is one that sends the fielder in the correct direction immediately. To do this, the angle of gaze must be allowed to increase (see Figure $8 b$ ).

The same argument applies if the ball is going to land behind the fielder. A fielder who maintains a constant angle of gaze will pass the place where the ball will land and have to run back to it (see Figure 9a). In contrast, a fielder who allows the angle of gaze to increase throughout the flight can arrive at the place to make the catch without overshooting it (see Figure 9b).

\section{Constant $d(\alpha) / d t$}

The analysis described above shows the importance of allowing $\alpha$ to increase. One way to accomplish this is to run so that $\mathrm{d}(\alpha) / \mathrm{dt}$ remains constant, that is, keeping $\mathrm{d}^{2}(\alpha) / \mathrm{dt}^{2}$ rather than $\mathrm{d}^{2}(\tan \alpha) / \mathrm{dt}^{2}$ at zero.

The problem with letting $\alpha$ increase is the danger that it will exceed $90^{\circ}$. Dienes and McLeod (1993) showed, for example, that allowing $\alpha$ to increase at constant velocity led to fielders running back too slowly to catch balls that were going over their heads on trajectories that we know, experimentally, skilled fielders would catch. Keeping $\mathrm{d}^{2}(\alpha) / \mathrm{dt}^{2}$ at zero allows $\alpha$ to exceed $90^{\circ}$ before the fielder reaches the place where the ball can be caught. Keeping $\mathrm{d}^{2}(\tan \alpha) / \mathrm{dt}^{2}$ at zero also allows $\alpha$ to increase. However, because $\tan \alpha$ grows more quickly than $\alpha$, the rate of increase slows down as $\alpha$ increases. Because $\tan \alpha$ grows more and more quickly as $\alpha$ approaches $90^{\circ}$, keeping $\mathrm{d}^{2}(\tan \alpha) / \mathrm{dt}^{2}$ at zero ensures that the rate of increase of $\alpha$ slows more and more as $\alpha$ increases. Thus, provided the fielder can run fast enough to keep $\mathrm{d}^{2}(\tan \alpha) / \mathrm{dt}^{2}$ at zero, this strategy ensures that $\alpha$ will never exceed $90^{\circ}$.

The way that the strategy followed by expert fielders allows $\alpha$ to increase but slows down the rate of increase as 

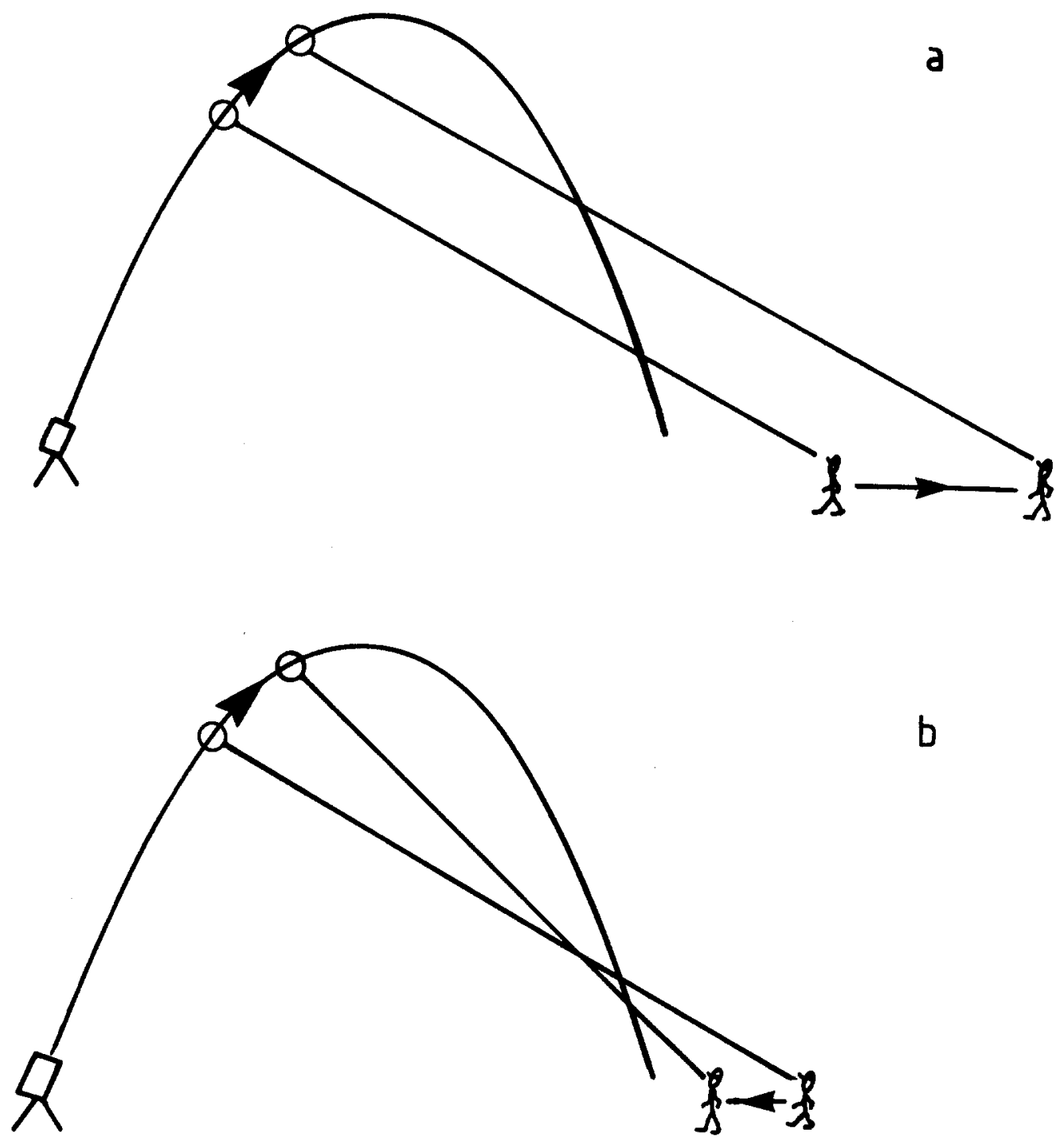

Figure 8. Two strategies for interception when the fielder should run forward. (a) Keeping the angle of elevation of gaze constant is a poor strategy because it can send the fielder in the wrong direction. (b) Allowing the angle of gaze to increase ensures that the fielder moves in the right direction.

$\alpha$ increases can be seen by plotting the value of $\alpha$ against time for the six catches shown in Figure 3. This is shown in Figure 10. The reduction in the rate of increase of $\alpha$ as $\alpha$ increases is demonstrated by the highly significant quadratic component to multiple regressions of $\alpha$ on $t(p<.001$ for each catch). This shows that fielders do not keep $d(\alpha) / d t$ constant as they run. Thus, as complex as the strategy of keeping $\mathrm{d}^{2}(\tan \alpha) / \mathrm{dt}^{2}$ at zero might seem, there are good reasons for both elements of it to have evolved.

\section{What Do Children Learn?}

Children who are learning to catch start by watching balls thrown toward them while standing still. Since $0^{\circ}<\alpha<$ $90^{\circ}$ will be true of all flights that land in their arms, and only those flights, it would not be surprising if they discovered the importance of this relationship. It seems reasonable to assume that they might try to use this fact when they have to start running for the ball. That is, they will try to keep $0^{\circ}<\alpha<90^{\circ}$ as they run.

But why should they discover that maintaining $d^{2}(\tan$ $\alpha) / \mathrm{dt}^{2}$ at zero is an efficient way of keeping $0^{\circ}<\alpha<90^{\circ}$ ? Extending the original observations of Chapman (1968) about watching parabolic flights, Dienes and McLeod (1993) showed that for a stationary fielder, at any point in the ball's flight and whatever its trajectory (i.e., independent of the effects of wind resistance), the sign of $\mathrm{d}^{2}(\tan \alpha) / \mathrm{dt}^{2}$ is almost always negative if the ball will land in front of the 

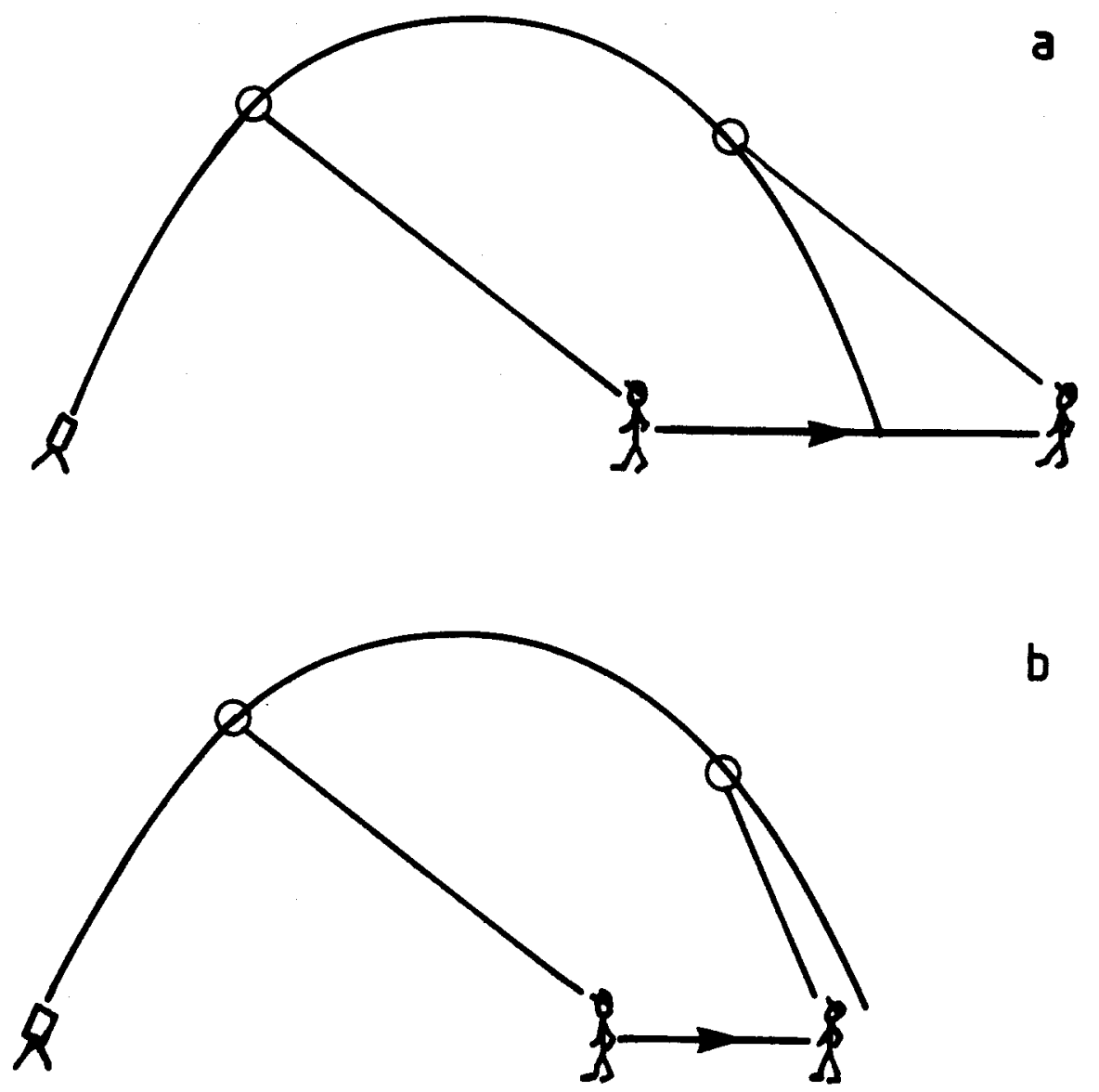

Figure 9. Two strategies for interception when the fielder is running backward. (a) Keeping the angle of elevation of gaze constant is an inefficient strategy because it will send the fielder past the point where the catch can be made. (b) Allowing the angle of gaze to increase avoids this problem.

observer and almost always positive if it will land behind the observer. ${ }^{9}$ So the fact that $\mathrm{d}^{2}(\tan \alpha) / \mathrm{dt}^{2}$ is correlated with the catchability of a ball flight is available to the stationary child. Whether mere exposure is sufficient to allow acquisition of this fact is a question that remains to be answered.

\section{Are People Really Computing $d^{2}(\tan \alpha) / d t^{2}$ ?}

Our data support those of Michaels and Oudejans (1992) in suggesting that competent catchers have discovered the effectiveness of keeping $d^{2}(\tan \alpha) / d^{2}$ at zero as the basis for an interception strategy. Of course, our data do not indicate how the computational problem of keeping $\mathrm{d}^{2}(\tan \alpha) / \mathrm{dt}^{2}$ at zero is solved. Skepticism about the conclusion might stem from the feeling that $\mathrm{d}^{2}(\tan \alpha) / \mathrm{dt}^{2}$ does not seem a particularly likely quantity for the nervous system to represent.

Todd (1981) showed that if participants knew the actual size of the ball and the acceleration due to gravity, zeroing an expression involving the second power of the optic size of the ball was equivalent to maintaining $\mathrm{d}^{2}(\tan \alpha) / \mathrm{dt}^{2}$ at zero in vacuo. Direct extension of Todd's analysis shows that the wind resistance problem can be overcome by using an expression involving the third power of optic size and the square of its first derivative. However, given that fielders start running almost immediately to catch balls thrown from distances of more than $50 \mathrm{~m}$, an approach requiring acute sensitivity to optic size seems implausible. It is possible that fielders do not compute $\tan \alpha$ at all. If they were to reduce a maintained value of $\mathrm{d}(\alpha) / \mathrm{dt}$ in a systematic way as $\alpha$ increased (as described in Dienes \& McLeod, 1993), this would keep $\mathrm{d}^{2}(\tan \alpha) / \mathrm{dt}^{2}$ at zero.

Another problem with the strategy of maintaining $d^{2}(\tan$ $\alpha) / \mathrm{dt}^{2}$ at zero is that the visual system is generally not particularly sensitive to acceleration. However, both Babler and Dannemiller (1993) and Tresilian (1995) have shown that the performance reported by us and by Michaels and Oudejans (1992) can be achieved by a system with the limitations of the human visual system.

\footnotetext{
${ }^{9}$ The limitations that lead to the qualifications almost and usually are described in detail in Dienes and McLeod (1993).
} 


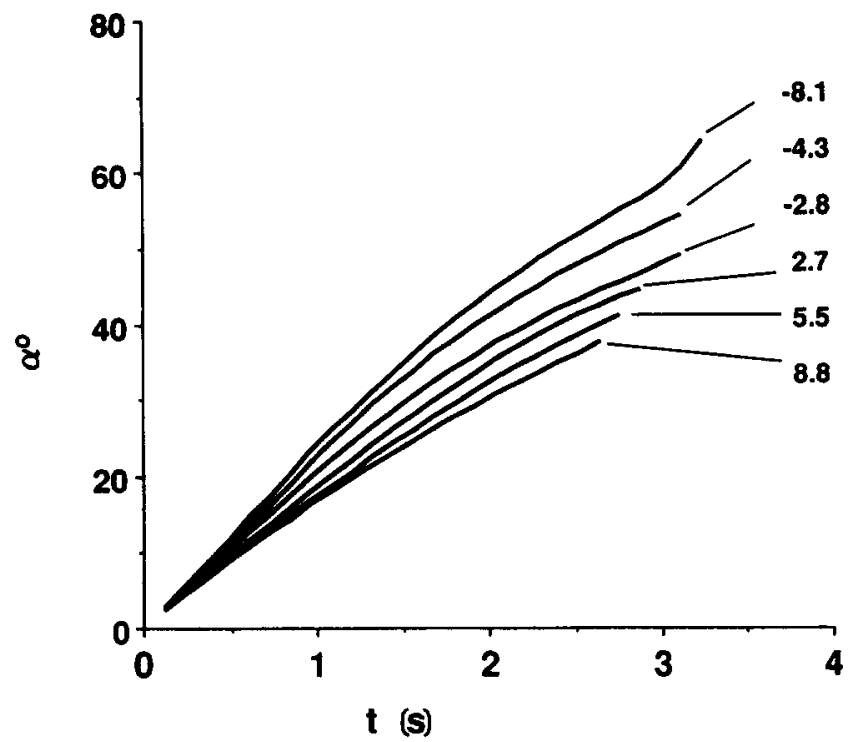

Figure 10. Angle of gaze as a function of time for the six catches shown in Figure 3. The numerical label shows how far (in meters) the catcher ran to make the catch. A negative sign indicates that he ran backward.

\section{References}

Babler, T. G., \& Dannemiller, J. L. (1993). Role of image acceleration in judging landing location of free-falling projectiles.
Journal of Experimental Psychology: Human Perception and Performance, 19, 15-31.

Brancazio, P. (1985). Looking into Chapman's homer: The physics of judging a flyball. American Journal of Physics, 53, 849-855.

Chapman, S. (1968). Catching a baseball. American Journal of Physics, 36, 868-870.

Daish, C. (1972). The physics of ball games. London: The English Universities Press.

Dienes, Z., \& McLeod, P. (1993). How to catch a cricket ball. Perception, 22, 1427-1439.

Michaels, C., \& Oudejans, R. (1992). The optics and actions of catching fly balls: Zeroing out optic acceleration. Ecological Psychology, 4, 199-222.

Regan, D. (1993). Binocular correlates of the direction of motion in depth. Vision Research, 33, 2359-2360.

Regan, D., Beverley, K., \& Cynader, M. (1979, July). The visual perception of motion in depth. Scientific American, 241, 122133.

Regan, D., \& Kanshal, S. (1994). Monocular discrimination of the direction of motion in depth. Vision Research, 34, 163-177.

Richards, V., \& Murphy, P. (1988). Viv Richards' cricket masterclass. London: Queen Anne Press.

Todd, J. (1981). Visual information about moving objects. Journal of Experimental Psychology: Human Perception and Performance, 7, 795-810.

Tresilian, J. (1995). Study of a servo-control strategy for projectile interception. Quarterly Journal of Experimental Psychology, 48, 688-715.

Received November 3, 1993

Revision received February 13, 1995

Accepted March 21, 1995

\section{Low Publication Prices for APA Members and Affiliates}

Keeping you up-to-date. All APA Fellows, Members, Associates, and Student Affiliates receive - as part of their annual dues-subscriptions to the American Psychologist and $A P A$ $M o n i t o r$. High School Teacher and International Affiliates receive subscriptions to the $A P A$ Monitor, and they may subscribe to the American Psychologist at a significantly reduced rate. In addition, all Members and Student Affiliates are eligible for savings of up to $60 \%$ (plus a journal credit) on all other APA journals, as well as significant discounts on subscriptions from cooperating societies and publishers (e.g., the American Association for Counseling and Development, Academic Press, and Human Sciences Press).

Essential resources. APA members and affiliates receive special rates for purchases of APA books, including the Publication Manual of the American Psychological Association, and on dozens of new topical books each year.

Other benefits of membership. Membership in APA also provides eligibility for competitive insurance plans, continuing education programs, reduced APA convention fees, and specialty divisions.

More information. Write to American Psychological Association, Membership Services, 750 First Street, NE, Washington, DC 20002-4242. 